\title{
Trudinger-Moser inequalities on the entire Heisenberg group
}

\author{
Yunyan Yang \\ Department of Mathematics, Renmin University of China, Beijing 100872, P. R. China
}

\begin{abstract}
Continuing our previous work (Cohn, Lam, Lu, Yang, Nonlinear Analysis (2011), doi: 10.1016 /j.na.2011.09.053), we obtain a class of Trudinger-Moser inequalities on the entire Heisenberg group, which indicate what the best constants are. All the existing proofs of similar inequalities on unbounded domain of the Euclidean space or the Heisenberg group are based on rearrangement argument. In this note, we propose a new approach to solve this problem. Specifically we get the global Trudinger-Moser inequality by gluing local estimates with the help of cut-off functions. Our method still works for similar problems when the Heisenberg group is replaced by the Eclidean space or complete noncompact Riemannian manifolds.
\end{abstract}

Key words: Trudinger-Moser inequality, singular Trudinger-Moser inequality, Adams inequality

2000 MSC: 46E35

\section{Introduction}

Let $\mathbb{H}^{n}=\mathbb{R}^{2 n} \times \mathbb{R}$ be the Heisenberg group whose group action is defined by

$$
(x, y, t) \circ\left(x^{\prime}, y^{\prime}, t^{\prime}\right)=\left(x+x^{\prime}, y+y^{\prime}, t+t^{\prime}+2\left(\left\langle y, x^{\prime}\right\rangle-\left\langle x, y^{\prime}\right\rangle\right)\right),
$$

where $x, y, x^{\prime}, y^{\prime} \in \mathbb{R}^{n}, t, t^{\prime} \in \mathbb{R}$, and $\langle\cdot, \cdot\rangle$ denotes the standard inner product in $\mathbb{R}^{n}$. Let us denote the parabolic dilation in $\mathbb{R}^{2 n} \times \mathbb{R}$ by $\delta_{\lambda}$, namely, $\delta_{\lambda}(\xi)=\left(\lambda x, \lambda y, \lambda^{2} t\right)$ for any $\xi=(x, y, t) \in \mathbb{R}^{2 n} \times \mathbb{R}$. The Jacobian determinant of $\delta_{\lambda}$ is $\lambda^{Q}$, where $Q=2 n+2$ is the homogeneous dimension of $\mathbb{H}^{n}$. The following norm

$$
|\xi|_{h}=\left[\left(\sum_{i=1}^{n}\left(x_{i}^{2}+y_{i}^{2}\right)\right)^{2}+t^{2}\right]^{\frac{1}{4}}
$$

is homogeneous of degree one with respect to the dilation $\delta_{\lambda}$. The associated distance between two points $\xi$ and $\eta$ of $\mathbb{H}^{n}$ is defined accordingly by

$$
d_{h}(\xi, \eta)=\left|\eta^{-1} \circ \xi\right|_{h},
$$

where $\eta^{-1}$ denotes the inverse of $\eta$ with respect to the group action, i.e. $\eta^{-1}=-\eta$. Obviously $d_{h}(\cdot, \cdot)$ is symmetric. The open ball of radius $r$ centered at $\xi$ is

$$
B_{h}(\xi, r)=\left\{\eta \in \mathbb{H}^{n}: d_{h}(\eta, \xi)<r\right\} .
$$

Email address: yunyanyang@ruc.edu.cn (Yunyan Yang) Preprint submitted to $* * *$ 
It is important to note that (see for example Stein [11], Section 5 of Chapter VIII)

$$
\left|B_{h}(\xi, r)\right|=\left|B_{h}(0, r)\right|=\left|B_{h}(0,1)\right| r^{Q},
$$

where $|\cdot|$ denotes the Lebesgue measure. The Lie algebra of $\mathbb{H}^{n}$ is generated by the left-invariant vector fields

$$
T=\frac{\partial}{\partial t}, X_{i}=\frac{\partial}{\partial x_{i}}+2 y_{i} \frac{\partial}{\partial t}, Y_{i}=\frac{\partial}{\partial y_{i}}-2 x_{i} \frac{\partial}{\partial t}, i=1, \cdots, n .
$$

These generators satisfy the non-commutative formula $\left[X_{i}, Y_{i}\right]=-4 \delta_{i j} T$. Denote by $\left|\nabla_{\mathbb{H}^{n}} u\right|$ the norm of the sub-elliptic gradient of a smooth function $u: \mathbb{H}^{n} \rightarrow \mathbb{R}:$

$$
\left|\nabla_{\mathbb{H}^{n} n} u\right|=\left(\sum_{i=1}^{n}\left(\left(X_{i} u\right)^{2}+\left(Y_{i} u\right)^{2}\right)\right)^{1 / 2} .
$$

Let $\Omega$ be an open set in $\mathbb{H}^{n}$. We use $W_{0}^{1, p}(\Omega)$ to denote the completion of $C_{0}^{\infty}(\Omega)$ under the norm

$$
\|u\|_{W_{0}^{1, p}(\Omega)}=\left(\int_{\Omega}\left(\left|\nabla_{\mathbb{H}^{n}} u\right|^{p}+|u|^{p}\right) d \xi\right)^{1 / p} .
$$

In [4], Cohn-Lu proved a Trudinger-Moser inequality on bounded smooth domains in the Hesenberg group $\mathbb{H}^{n}$. Precisely, there exists some constant $C_{n}$ depending only on $n$ such that for all bounded smooth domain $\Omega \subset \mathbb{H}^{n}$, if $u \in W_{0}^{1, Q}(\Omega)$ satisfies $\left\|\nabla_{\mathbb{H}^{n}} u\right\|_{L^{Q}(\Omega)} \leq 1$, then

$$
\int_{\Omega} e^{\alpha_{Q}|u|^{\left.\right|^{\prime}}} d \xi \leq C_{n}|\Omega|
$$

where $Q^{\prime}=Q /(Q-1), \alpha_{Q}=Q \sigma_{Q}^{1 /(Q-1)}, \sigma_{Q}=\Gamma\left(\frac{1}{2}\right) \Gamma\left(n+\frac{1}{2}\right) \omega_{2 n-1} / n !, \omega_{2 n-1}$ is the surface area of the unit sphere in $\mathbb{R}^{2 n}$. Furthermore, the integrals of all $u \in W_{0}^{1, Q}(\Omega)$ satisfying $\left\|\nabla_{\mathbb{H}^{n}} u\right\|_{L^{Q}(\Omega)} \leq 1$ are not uniformly bounded if $\alpha_{Q}$ is replaced by any larger number. Recently, Cohn, Lam, Lu and the author [3] obtained a Trudinger-Moser inequality on the Heisenberg group $\mathbb{H}^{n}$. Note that $W^{1, Q}\left(\mathbb{H}^{n}\right)$ is the completion of $C_{0}^{\infty}\left(\mathbb{H}^{n}\right)$ under the norm (1.6) with $\Omega$ replaced by $\mathbb{H}^{n}$. We have the following:

Theorem A ([3]). There exists some constant $\alpha^{*}: 0<\alpha^{*} \leq \alpha_{Q}$ such that for any pair $\beta$ and $\alpha$ satisfying $0 \leq \beta<Q, 0<\alpha \leq \alpha^{*}$, and $\frac{\alpha}{\alpha^{*}}+\frac{\beta}{Q} \leq 1$, there holds

$$
\sup _{\|u\|_{W^{1}, Q \mathbb{H}^{n}} \leq 1} \int_{\mathbb{H}^{n}} \frac{1}{|\xi|_{h}^{\beta}}\left\{e^{\alpha|u|^{Q^{\prime}}}-\sum_{k=0}^{Q-2} \frac{\alpha^{k}|u|^{k Q^{\prime}}}{k !}\right\} d \xi<\infty .
$$

When $\frac{\alpha}{\alpha^{*}}+\frac{\beta}{Q}>1$, the integral in $(1.8)$ is still finite for any $u \in W^{1, Q}\left(\mathbb{H}^{n}\right)$, but the supremum is infinite if further $\frac{\alpha}{\alpha_{Q}}+\frac{\beta}{Q}>1$.

Theorem A is an analogue of (Adimurthi-Yang [1], Theorem 1.1). Earlier works on this topic (Trudinger-Moser inequalities on unbounded domain of $\mathbb{R}^{n}$ ) were done by Cao [2], Panda [9], do Ó [5], Ruf [10], Li-Ruf [8] and others. The proof of Theorem A is based on symmetrization argument, radial lemma and the Young inequality. Note that $\alpha^{*}$ in Theorem A is not explicitly 
known. A natural question is what the best constant $\alpha$ for 1.8 is. Denote an equivalent norm in $W^{1, Q}\left(\mathbb{H}^{n}\right)$ by

$$
\|u\|_{1, \tau}=\left(\int_{\mathbb{H}^{n}}\left(\left|\nabla_{\mathbb{H}^{n}} u\right|^{Q}+\tau|u|^{Q}\right) d \xi\right)^{\frac{1}{Q}}
$$

for any fixed number $\tau>0$. Our main result is the following:

Theorem 1.1. Let $\tau$ be any positive real number. Let $Q, Q^{\prime}$ and $\alpha_{Q}$ be as in (1.7). For any $\beta: 0 \leq \beta<Q$ and $\alpha: 0<\alpha<\alpha_{Q}(1-\beta / Q)$, there holds

$$
\sup _{\|u\|_{1, \tau} \leq 1} \int_{\mathbb{H}^{n}} \frac{1}{|\xi|_{h}^{\beta}}\left\{e^{\alpha|u|^{Q^{\prime}}}-\sum_{k=0}^{Q-2} \frac{\alpha^{k}|u|^{k Q^{\prime}}}{k !}\right\} d \xi<\infty
$$

When $\alpha>\alpha_{Q}(1-\beta / Q)$, the above integral is still finite for any $u \in W^{1, Q}\left(\mathbb{H}^{n}\right)$, but the supremum is infinite.

Clearly Theorem 1.1 implies that the best constant for the inequality $(1.10)$ is $\alpha_{Q}(1-\beta / Q)$. But we do not know whether or not (1.10) still holds when $\alpha=\alpha_{Q}(1-\beta / Q)$. Even so, 1.10 gives more information than (1.8).

According to the author's knowledge, the existing proofs of Trudinger-Moser inequalities for unbounded domains are all based on the rearrangement theory [6]. It is not known that whether or not this technique can be successfully applied to the Heisenberg group case.

To prove Theorem 1.1, we propose a new approach. The idea can be described as follows. Firstly, using (1.7), we derive a local Trudinger-Moser inequality, namely, for any fixed $r>0$ and all $\xi_{0} \in \mathbb{H}^{n}$, there exists some constant $C$ depending only on $n, r$ and $\beta$ such that

$$
\int_{B_{h}\left(\xi_{0}, r\right)} \frac{1}{|\xi|_{h}^{\beta}}\left\{e^{\alpha|u|^{Q^{\prime}}}-\sum_{k=0}^{Q-2} \frac{\alpha^{k}|u|^{k Q^{\prime}}}{k !}\right\} d \xi \leq C \int_{B_{h}\left(\xi_{0}, r\right)}\left|\nabla_{\mathbb{H}^{n}} u\right|^{Q} d \xi
$$

provided that $0 \leq \alpha<\alpha_{Q}(1-\beta / Q)$ and $\int_{B_{h}\left(\xi_{0}, r\right)}\left|\nabla_{\mathbb{H}^{n}} u\right|^{Q} d \xi \leq 1$. Secondly, fixing sufficiently large $r>0$, we select a specific sequence of Heisenberg balls $\left\{B_{h}\left(\xi_{i}, r\right)\right\}_{i=1}^{\infty}$ to cover the Heisenberg group $\mathbb{H}^{n}$. Then we choose appropriate cut-off function $\phi_{i}$ on each $B_{h}\left(\xi_{i}, r\right)$. Finally, we obtain (1.10) by gluing all local estimates (1.11) for $\phi_{i} u$. We remark that our method still works for similar problems when the Heisenberg group is replaced by the Eclidean space or complete noncompact Riemannian manifolds. In the Eclidean space case, $\tau$ can also be arbitrary in (1.10). But in the manifold case, the choice of $\tau$ may depend on the geometric structure (see [13], Theorem 2.3). As an easy consequence of Theorem 1.1 (in fact a special case $\beta=0$ ), the following corollary holds.

Corollary 1.2. Let $Q=2 n+2$. For any $q \geq Q, W^{1, Q}\left(\mathbb{H}^{n}\right)$ is continuously embedded in $L^{q}\left(\mathbb{H}^{n}\right)$.

The remaining part of this note is organized as follows. In section 2, we prove a covering lemma for $\mathbb{H}^{n}$; Cut-off functions are selected for the subsequent analysis in section 3 ; The proof of Theorem 1.1 is completed in section 4. 


\section{A covering lemma for the Heisenberg group}

In this section, we will use a sequence of Heisenberg balls with the same radius to cover the entire Heisenberg group $\mathbb{H}^{n}$. We require these balls to satisfy the following properties: $(i)$ For any $\xi \in \mathbb{H}^{n}$, $\xi$ belongs to at most $N$ balls for some constant integer $N$ which is independent of the base point $\xi$; (ii) If the radius of those balls becomes appropriately smaller, then they are disjoint.

Firstly, we need to understand the Heisenberg distance between two points of the Heisenberg group $\mathbb{H}^{n}$. The following two properties are more or less standard. We prefer to present them by our own way.

Proposition 2.1. Let $\xi$ and $\eta$ be two points of $\mathbb{H}^{n}$. There holds

$$
\left|\eta^{-1} \circ \xi\right|_{h} \leq 3\left(|\xi|_{h}+|\eta|_{h}\right)
$$

where $|\cdot|_{h}$ is the homogeneous norm defined by (1.2).

Proof. Write $\xi=(x, y, t), \eta=\left(x^{\prime}, y^{\prime}, t^{\prime}\right)$. Then (1.1) gives

$$
\eta^{-1} \circ \xi=\left(x-x^{\prime}, y-y^{\prime}, t-t^{\prime}-2\left(\left\langle y, x^{\prime}\right\rangle-\left\langle x, y^{\prime}\right\rangle\right)\right) .
$$

Since $\left(\left|x-x^{\prime}\right|^{2}+\left|y-y^{\prime}\right|^{2}\right)^{1 / 2} \leq\left(|x|^{2}+|y|^{2}\right)^{1 / 2}+\left(\left|x^{\prime}\right|^{2}+\left|y^{\prime}\right|^{2}\right)^{1 / 2}$ and

$$
\left|2\left(\left\langle y, x^{\prime}\right\rangle-\left\langle x, y^{\prime}\right\rangle\right)\right| \leq|x|^{2}+|y|^{2}+\left|x^{\prime}\right|^{2}+\left|y^{\prime}\right|^{2},
$$

we have by using the inequality $\sqrt{a+b} \leq \sqrt{a}+\sqrt{b}(a \geq 0, b \geq 0)$ repeatedly

$$
\begin{aligned}
\left|\eta^{-1} \circ \xi\right|_{h} & =\left[\left(\sum_{i=1}^{n}\left(\left(x_{i}-x_{i}^{\prime}\right)^{2}+\left(y_{i}-y_{i}^{\prime}\right)^{2}\right)\right)^{2}+\left(t-t^{\prime}-2\left(\left\langle y, x^{\prime}\right\rangle-\left\langle x, y^{\prime}\right\rangle\right)\right)^{2}\right]^{\frac{1}{4}} \\
& \leq\left(\sum_{i=1}^{n}\left(\left(x_{i}-x_{i}^{\prime}\right)^{2}+\left(y_{i}-y_{i}^{\prime}\right)^{2}\right)\right)^{\frac{1}{2}}+\left|t-t^{\prime}-2\left(\left\langle y, x^{\prime}\right\rangle-\left\langle x, y^{\prime}\right\rangle\right)\right|^{\frac{1}{2}} \\
& \leq 2\left(\sum_{i=1}^{n}\left(x_{i}^{2}+y_{i}^{2}\right)\right)^{\frac{1}{2}}+2\left(\sum_{i=1}^{n}\left(x_{i}^{\prime 2}+y_{i}^{\prime 2}\right)\right)^{\frac{1}{2}}+|t|^{\frac{1}{2}}+\left|t^{\prime}\right|^{\frac{1}{2}} \\
& \leq 3\left(|\xi|_{h}+|\eta|_{h}\right) .
\end{aligned}
$$

Proposition 2.2. Let $\xi, \eta$, $\zeta$ be arbitrary points of $\mathbb{H}^{n}$. Then we have

$$
d_{h}(\xi, \eta) \leq 3\left(d_{h}(\xi, \zeta)+d_{h}(\zeta, \eta)\right)
$$

where $d_{h}(\cdot, \cdot)$ is the distance function defined by (1.3).

Proof. Note that $\left|\gamma^{-1}\right|_{h}=|\gamma|_{h}$ for all $\gamma \in \mathbb{H}^{n}$. It follows from Proposition 2.1 that

$$
\begin{aligned}
d_{h}(\xi, \eta) & =\left|\eta^{-1} \circ \xi\right|_{h} \\
& =\left|\eta^{-1} \circ \zeta \circ \zeta^{-1} \circ \xi\right|_{h} \\
& \leq 3\left(\left|\eta^{-1} \circ \zeta\right|_{h}+\left|\zeta^{-1} \circ \xi\right|_{h}\right) \\
& =3\left(d_{h}(\zeta, \eta)+d_{h}(\xi, \zeta)\right) .
\end{aligned}
$$


This gives the desired result.

Secondly, by adapting an argument of (Hebey [7], Lemma 1.6), we obtain the following useful covering lemma.

Lemma 2.3. Let $\rho>0$ be given. There exists a sequence $\left(\xi_{i}\right)$ of points of $\mathbb{H}^{n}$ such that for any $r \geq \rho$ :

(i) $\cup_{i} B_{h}\left(\xi_{i}, \rho\right)=\mathbb{H}^{n}$ and for any $i \neq j, B_{h}\left(\xi_{i}, \rho / 6\right) \cap B_{h}\left(\xi_{j}, \rho / 6\right)=\varnothing$;

(ii) for any $\xi \in \mathbb{H}^{n}$, $\xi$ belongs to at most $\left[(24 r / \rho)^{Q}\right]$ balls $B_{h}\left(\xi_{i}, r\right)$, where $\left[(24 r / \rho)^{Q}\right]$ denotes the integral part of $(24 r / \rho)^{Q}$.

Proof. Firstly, we claim that there exists a sequence $\left(\xi_{i}\right)$ of points of $\mathbb{H}^{n}$ such that

$$
\cup_{i} B_{h}\left(\xi_{i}, \rho\right)=\mathbb{H}^{n} \text { and } \forall i \neq j, B_{h}\left(\xi_{i}, \rho / 6\right) \cap B_{h}\left(\xi_{j}, \rho / 6\right)=\varnothing .
$$

To see this, we set

$$
X_{\rho}=\left\{\text { sequence }\left(\xi_{i}\right)_{i \in I}: \xi_{i} \in \mathbb{H}^{n}, I \text { is countable and } \forall i \neq j, d_{h}\left(\xi_{i}, \xi_{j}\right) \geq \rho\right\} .
$$

Then $X_{\rho}$ is partially ordered by inclusion and every element in $X_{\rho}$ has an upper bound in the sense of inclusion. Hence, by Zorn's lemma, $X_{\rho}$ contains a maximal element $\left(\xi_{i}\right)_{i \in I}$. On one hand, if $\cup_{i} B_{h}\left(\xi_{i}, \rho\right) \neq \mathbb{H}^{n}$, then there exists a point $\xi \in \mathbb{H}^{n}$ such that $d_{h}\left(\xi_{i}, \xi\right) \geq \rho$ for all $i \in$ I. This contradicts the maximality of $\left(\xi_{i}\right)_{i \in I}$. Hence $\cup_{i} B_{h}\left(\xi_{i}, \rho\right)=\mathbb{H}^{n}$. On the other hand, if $B_{h}\left(\xi_{i}, \rho / 6\right) \cap B_{h}\left(\xi_{j}, \rho / 6\right) \neq \varnothing$ for some $i \neq j$, then we can take some $\eta \in B_{h}\left(\xi_{i}, \rho / 6\right) \cap B_{h}\left(\xi_{j}, \rho / 6\right)$. It follows from Proposition 2.2 that

$$
\begin{aligned}
d_{h}\left(\xi_{i}, \xi_{j}\right) & \leq 3\left(d_{h}\left(\xi_{i}, \eta\right)+d_{h}\left(\eta, \xi_{j}\right)\right) \\
& <3\left(\frac{\rho}{6}+\frac{\rho}{6}\right)=\rho .
\end{aligned}
$$

This contradicts the fact that $d_{h}\left(\xi_{i}, \xi_{j}\right) \geq \rho$ for any $i \neq j$. Thus our claim (2.1) holds.

Assume $\left(\xi_{i}\right)$ satisfies (2.1). For any fixed $r>0$ and $\xi \in \mathbb{H}^{n}$ we set

$$
I_{r}(\xi)=\left\{i \in I: \xi \in B_{h}\left(\xi_{i}, r\right)\right\} .
$$

By (1.4) and Proposition 2.2, we have for $r \geq \rho$

$$
\begin{aligned}
\left|B_{h}(\xi, r)\right| & =4^{-Q}\left|B_{h}(\xi, 4 r)\right| \\
& \geq 4^{-Q} \sum_{i \in I_{r}(\xi)}\left|B_{h}\left(\xi_{i}, \rho / 6\right)\right| \\
& =4^{-Q} \operatorname{Card} I_{r}(\xi)(\rho / 6)^{Q}\left|B_{h}(0,1)\right|,
\end{aligned}
$$

where Card $I_{r}(\xi)$ denotes the cardinality of the set $I_{r}(\xi)$. As a consequence, for $r \geq \rho$ there holds

$$
\text { Card } I_{r}(\xi) \leq(24 r / \rho)^{Q} \text {. }
$$

This completes the proof of the lemma. 


\section{Cut-off functions on Heisenberg balls}

In this section, we will construct cut-off functions on Heisenberg balls. To do this, we first estimate the gradient of the distance function as follows.

Lemma 3.1. Let $\xi_{0}$ be any fixed point of $\mathbb{H}^{n}$. Define a function $\rho(\xi)=d_{h}\left(\xi, \xi_{0}\right)$. Then we have $\left|\nabla_{\mathbb{H}^{n}} \rho(\xi)\right| \leq 1$ for any $\xi \neq \xi_{0}$.

Proof. Write $\xi=\left(x_{1}, \cdots, x_{n}, y_{1}, \cdots, y_{n}, t\right)$ and $\xi_{0}=\left(x_{01}, \cdots, x_{0 n}, y_{01}, \cdots, y_{0 n}, t_{0}\right)$. For any $\xi \neq \xi_{0}$, we set

$$
E=\sum_{i=1}^{n}\left(\left(x_{i}-x_{0 i}\right)^{2}+\left(y_{i}-y_{0 i}\right)^{2}\right), \quad F=t-t_{0}-2 \sum_{i=1}^{n}\left(x_{i} y_{0 i}-y_{i} x_{0 i}\right) .
$$

Then by (1.1) and (1.3),

$$
\rho(\xi)=\left|\xi_{0}^{-1} \circ \xi\right|_{h}=\left(E^{2}+F^{2}\right)^{1 / 4} .
$$

We calculate

$$
\frac{\partial}{\partial x_{i}} \rho=\rho^{-3}\left(\left(x_{i}-x_{0 i}\right) E-y_{0 i} F\right), \quad 2 y_{i} \frac{\partial}{\partial t} \rho=\rho^{-3} y_{i} F,
$$

and then by (1.5),

$$
X_{i} \rho=\frac{\partial}{\partial x_{i}} \rho+2 y_{i} \frac{\partial}{\partial t} \rho=\rho^{-3}\left(\left(x_{i}-x_{0 i}\right) E+\left(y_{i}-y_{0 i}\right) F\right) .
$$

Similarly we have

$$
\frac{\partial}{\partial y_{i}} \rho=\rho^{-3}\left(\left(y_{i}-y_{0 i}\right) E+x_{0 i} F\right)
$$

and thus by $(1.5)$,

$$
Y_{i} \rho=\frac{\partial}{\partial y_{i}} \rho-2 x_{i} \frac{\partial}{\partial t} \rho=\rho^{-3}\left(\left(y_{i}-y_{0 i}\right) E+\left(x_{0 i}-x_{i}\right) F\right) .
$$

It follows that

$$
\left(X_{i} \rho\right)^{2}+\left(Y_{i} \rho\right)^{2}=\rho^{-6}\left(\left(y_{i}-y_{0 i}\right)^{2}+\left(x_{i}-x_{0 i}\right)^{2}\right)\left(E^{2}+F^{2}\right) .
$$

Note that $E^{2}+F^{2}=\rho^{4}$. We obtain

$$
\begin{aligned}
\left|\nabla_{\mathbb{H}^{n}} \rho\right| & =\left(\sum_{i=1}^{n}\left(\left(X_{i} \rho\right)^{2}+\left(Y_{i} \rho\right)^{2}\right)\right)^{1 / 2} \\
& =\rho^{-3} E^{1 / 2}\left(E^{2}+F^{2}\right)^{1 / 2} \\
& =\rho^{-1} E^{1 / 2} \leq 1 .
\end{aligned}
$$

This completes the proof of the lemma. 
Now we construct cut-off functions. Let $\phi: \mathbb{R} \rightarrow \mathbb{R}$ be a smooth function such that $0 \leq \phi \leq 1$, $\phi \equiv 1$ on the interval $[-1,1], \phi \equiv 0$ on $(-\infty,-2) \cup(2, \infty)$, and $\left|\phi^{\prime}(t)\right| \leq 2$ for all $t \in \mathbb{R}$. Let $r>0$ be given. Define a function on $\mathbb{H}^{n}$ by

$$
\phi_{0}(\xi)=\phi\left(\frac{d_{h}\left(\xi, \xi_{0}\right)}{r}\right) .
$$

Then $\phi_{0}$ is a cut-off function supported on the Heisenberg ball $B_{h}\left(\xi_{0}, 2 r\right)$. The estimate of the gradient of $\phi_{0}$ is very important for the subsequent analysis. Precisely we have the following:

Lemma 3.2. For any fixed $r>0$ and $\xi_{0} \in \mathbb{H}^{n}$, let $\phi_{0}$ be defined by (3.1). Then $\phi_{0}$ is supported in $B_{h}\left(\xi_{0}, 2 r\right), 0 \leq \phi_{0} \leq 1, \phi_{0} \equiv 1$ on $B_{h}\left(\xi_{0}, r\right)$, and $\left|\nabla_{\mathbb{H}^{n}} \phi_{0}(\xi)\right| \leq 2 / r$ for all $\xi \in \mathbb{H}^{n}$.

Proof. We only need to explain the last assertion, namely $\left|\nabla_{\mathbb{H}^{n}} \phi_{0}(\xi)\right| \leq 2 / r$ for all $\xi \in \mathbb{H}^{n}$. Since $\phi_{0} \equiv 1$ on $B_{h}\left(\xi_{0}, r\right)$, we have $\nabla_{\mathbb{H}^{n}} \phi_{0} \equiv 0$ on $B_{h}\left(\xi_{0}, r\right)$, particularly $\nabla_{\mathbb{H}^{n}} \phi_{0}(0)=0$. For $\xi \neq \xi_{0}$, a simple calculation shows

$$
\nabla_{\mathbb{H}^{n}} \phi_{0}(\xi)=\frac{1}{r} \phi^{\prime} \nabla_{\mathbb{H}^{n}} d_{h}\left(\xi, \xi_{0}\right) .
$$

This together with Lemma 3.1 and $\left|\phi^{\prime}\right| \leq 2$ concludes the last assertion.

\section{Proof of Theorem 1.1}

In this section, we will prove Theorem 1.1. For simplicity, we define a smooth function $\zeta: \mathbb{N} \times \mathbb{R} \rightarrow \mathbb{R}$ by

$$
\zeta(m, s)=e^{s}-\sum_{k=0}^{m-2} \frac{s^{k}}{k !}, \quad \forall m \geq 2 .
$$

As we promised in the introduction, we first derive a local Trudinger-Moser inequality for the Heisenberg group $\mathbb{H}^{n}$ by using (1.7). Let $Q, Q^{\prime}$ and $\alpha_{Q}$ be given by (1.7). Then we have the following:

Lemma 4.1. Let $r>0$ be given and $\xi_{0}$ be any point of $\mathbb{H}^{n}$. If $0 \leq \beta<Q, 0 \leq \alpha \leq \alpha_{Q}(1-$ $\beta / Q)$, and $w \in W_{0}^{1, Q}\left(B_{h}\left(\xi_{0}, r\right)\right)$ satisfies $\int_{B_{h}\left(\xi_{0}, r\right)}\left|\nabla_{\mathbb{H}^{n}} w\right|^{Q} d \xi \leq 1$, then there exists some constant $C$ depending only on $n, r$ and $\beta$ such that

$$
\int_{B_{h}\left(\xi_{0}, r\right)} \frac{1}{|\xi|_{h}^{\beta}} \zeta\left(Q, \alpha|w|^{Q^{\prime}}\right) d \xi \leq C \int_{B_{h}\left(\xi_{0}, r\right)}\left|\nabla_{\mathbb{H}^{n}} w\right|^{Q} d \xi .
$$

Proof. Using Proposition 2.2, we have that

$$
\left|\xi_{0}\right|_{h} \leq 3\left(d_{h}\left(\xi, \xi_{0}\right)+|\xi|_{h}\right), \quad \forall \xi \in \mathbb{H} .
$$

If $\left|\xi_{0}\right|_{h}>6 r$, then for any $\xi \in B_{h}\left(\xi_{0}, r\right)$ there holds

$$
|\xi|_{h} \geq \frac{\left|\xi_{0}\right|_{h}}{3}-d_{h}\left(\xi, \xi_{0}\right)>r .
$$


Let $\widetilde{w}=w /\left\|\nabla_{\mathbb{H}^{n}} w\right\|_{L^{Q}\left(B_{h}\left(\xi_{0}, r\right)\right)}$. Since $\left\|\nabla_{\mathbb{H}^{n}} w\right\|_{L^{Q}\left(B_{h}\left(\xi_{0}, r\right)\right)} \leq 1$ and $0 \leq \alpha \leq \alpha_{Q}(1-\beta / Q)$, we have

$$
\begin{aligned}
\zeta\left(Q, \alpha|w|^{Q^{\prime}}\right) & =\sum_{k=Q-1}^{\infty} \frac{\alpha^{k}|w|^{Q^{\prime} k}}{k !} \\
& =\sum_{k=Q-1}^{\infty} \frac{\alpha^{k}|| \nabla_{\mathbb{H}^{n}} w \|_{L^{Q}\left(B_{h}\left(\xi_{0}, r\right)\right.}^{\left.Q^{\prime}\right)}|\widetilde{w}|^{Q^{\prime} k}}{k !} \\
& \leq\left\|\nabla_{\mathbb{H}^{n}} w\right\|_{L^{Q}\left(B_{h}\left(\xi_{0}, r\right)\right)^{Q}}^{Q} \zeta\left(Q, \alpha|\widetilde{w}|^{Q^{\prime}}\right) .
\end{aligned}
$$

By (1.4) and (1.7),

$$
\int_{B_{h}\left(\xi_{0}, r\right)} \zeta\left(Q, \alpha_{Q}|\widetilde{w}|^{Q^{\prime}}\right) d \xi \leq C_{n} r^{Q}\left|B_{h}(0,1)\right|,
$$

where $C_{n}$ is given by (1.7). Hence when $\left|\xi_{0}\right|_{h}>6 r$ and $0 \leq \alpha \leq \alpha_{Q}(1-\beta / Q)$, we have by using (4.3) and (4.4),

$$
\begin{aligned}
\int_{B_{h}\left(\xi_{0}, r\right)} \frac{1}{\mid \xi \xi_{h}^{\beta}} \zeta\left(Q, \alpha|w|^{Q^{\prime}}\right) d \xi & \leq r^{-\beta} \int_{B_{h}\left(\xi_{0}, r\right)} \zeta\left(Q, \alpha|w|^{Q^{\prime}}\right) d \xi \\
& \leq C_{n} r^{Q-\beta}\left|B_{h}(0,1)\right| \int_{B_{h}\left(\xi_{0}, r\right)}\left|\nabla_{\mathbb{H}^{n}} w\right|^{Q} d \xi .
\end{aligned}
$$

In the following we assume $\left|\xi_{0}\right|_{h} \leq 6 r$. If $\xi \in B_{h}\left(\xi_{0}, r\right)$, then Proposition 2.2 implies that

$$
|\xi|_{h} \leq 3\left(d_{h}\left(\xi, \xi_{0}\right)+\left|\xi_{0}\right|_{h}\right)<21 r .
$$

Hölder's inequality together with 1.7 implies that there exits some constant $\widetilde{C}$ depending only on $n, r$ and $\beta$ such that

$$
\int_{B_{h}\left(\xi_{0}, r\right)} \frac{1}{|\xi|_{h}^{\beta}} \zeta\left(Q, \alpha|\widetilde{w}|^{Q^{\prime}}\right) d \xi \leq \int_{|\xi| h \leq 21 r} \frac{1}{|\xi|_{h}^{\beta}} \zeta\left(Q, \alpha|\widetilde{w}|^{Q^{\prime}}\right) d \xi \leq \widetilde{C} .
$$

It then follows from (4.4) that

$$
\begin{aligned}
\int_{B_{h}\left(\xi_{0}, r\right)} \frac{1}{|\xi|_{h}^{\beta}} \zeta\left(Q,\left.\alpha|w|\right|^{Q^{\prime}}\right) d \xi & \leq\left\|\nabla_{\mathbb{H}^{n}} w\right\|_{L^{Q}\left(B_{h}\left(\xi_{0}, r\right)\right)}^{Q} \int_{B_{h}\left(\xi_{0}, r\right)} \frac{1}{|\xi|_{h}^{\beta}} \zeta\left(Q, \alpha|\widetilde{w}|^{Q^{\prime}}\right) d \xi \\
& \leq \widetilde{C} \int_{B_{h}\left(\xi_{0}, r\right)}\left|\nabla_{\mathbb{H}^{n} n} w\right|^{Q} d \xi .
\end{aligned}
$$

Hence (4.2) holds.

Proof of Theorem 1.1. Firstly, we prove (1.10). Let $\tau>0$ and $\alpha: 0 \leq \alpha<\alpha_{Q}(1-\beta / Q)$ be fixed. Since $C_{0}^{\infty}\left(\mathbb{H}^{n}\right)$ is dense in $W^{1, Q}\left(\mathbb{H}^{n}\right)$ under the norm (1.9), it suffices to prove (1.10) for all $u \in C_{0}^{\infty}\left(\mathbb{H}^{n}\right)$ with

$$
\int_{\mathbb{H}^{n}}\left(\left|\nabla_{\mathbb{H}^{n}} u\right|^{Q}+\tau|u|^{Q}\right) d \xi \leq 1 .
$$

Assume $u \in C^{\infty}\left(\mathbb{H}^{n}\right)$ satisfies 4.5 . Let $r>0$ be a sufficiently large number to be determined later. By Lemma 2.3, there exists a sequence $\left(\xi_{i}\right)$ of points of $\mathbb{H}^{n}$ such that

$$
\cup_{i} B_{h}\left(\xi_{i}, r\right)=\mathbb{H}^{n} \text { and } \forall i \neq j, B_{h}\left(\xi_{i}, r / 6\right) \cap B_{h}\left(\xi_{j}, r / 6\right)=\varnothing,
$$


and for any $\xi \in \mathbb{H}^{n}$,

$$
\xi \text { belongs to at most } 48^{Q} \text { balls } B_{h}\left(\xi_{i}, 2 r\right) \text {. }
$$

Let $\phi$ be a smooth function given by (3.1). For each $\xi_{i}$, we set

$$
\phi_{i}(\xi)=\phi\left(\frac{d_{h}\left(\xi, \xi_{i}\right)}{r}\right), \quad \forall \xi \in \mathbb{H}^{n} .
$$

It follows from Lemma 3.2 that $0 \leq \phi_{i} \leq 1, \phi_{i} \equiv 1$ on $B_{h}\left(\xi_{i}, r\right), \phi_{i} \equiv 0$ outside $B_{h}\left(\xi_{i}, 2 r\right)$, and

$$
\left|\nabla_{\mathbb{H}^{n}} \phi_{i}(\xi)\right| \leq \frac{2}{r}, \forall \xi \in \mathbb{H}^{n} .
$$

Clearly $\phi_{i}^{2} u \in W_{0}^{1, Q}\left(B_{h}\left(\xi_{i}, 2 r\right)\right)$. Since $u$ satisfies (4.5), we have that

$$
\int_{\mathbb{H}^{n}}\left|\nabla_{\mathbb{H}^{n}} u\right|^{Q} d \xi \leq 1, \text { and } \int_{\mathbb{H}^{n}}|u|^{Q} d \xi \leq \frac{1}{\tau}
$$

Minkowski inequality together with (4.8) and $0 \leq \phi_{i} \leq 1$ leads to

$$
\begin{aligned}
\left(\int_{B_{h}\left(\xi_{i}, 2 r\right)}\left|\nabla_{\mathbb{H}^{n}}\left(\phi_{i}^{2} u\right)\right|^{Q} d \xi\right)^{1 / Q} & \leq\left(\int_{B_{h}\left(\xi_{i}, 2 r\right)} \phi_{i}^{2 Q}\left|\nabla_{\mathbb{H}^{n}} u\right|^{Q} d \xi\right)^{1 / Q}+\left(\int_{B_{h}\left(\xi_{i}, 2 r\right)}\left|\nabla_{\mathbb{H}^{n}} \phi_{i}^{2}\right|^{Q}|u|^{Q} d \xi\right)^{1 / Q} \\
& \leq\left(\int_{B_{h}\left(\xi_{i}, 2 r\right)}\left|\nabla_{\mathbb{H}^{n}} u\right|^{Q} d \xi\right)^{1 / Q}+\frac{4}{r}\left(\int_{B_{h}\left(\xi_{i}, 2 r\right)}|u|^{Q} d \xi\right)^{1 / Q} \\
& \leq 1+\frac{4}{\tau r} .
\end{aligned}
$$

Define $\widetilde{u}_{i}=\phi_{i}^{2} u /\left(1+\frac{4}{\tau r}\right)$. Then $\widetilde{u}_{i} \in W_{0}^{1, Q}\left(B_{h}\left(\xi_{i}, 2 r\right)\right)$ and $\int_{B_{h}\left(\xi_{i}, 2 r\right)}\left|\nabla_{\mathbb{H}^{n}} \widetilde{u}_{i}\right|^{Q} d \xi \leq 1$. Since $\alpha<$ $\alpha_{Q}(1-\beta / Q)$, we can select $r$ sufficiently large such that

$$
\alpha\left(1+\frac{4}{\tau r}\right)^{Q^{\prime}}<\alpha_{Q}(1-\beta / Q) .
$$

This together with Lemma 4.1 implies that there exists some constant $C$ depending only on $n, r$ and $\beta$ such that

$$
\begin{aligned}
\int_{B_{h}\left(\xi_{i}, 2 r\right)} \frac{1}{\mid \xi^{\beta}} \zeta\left(Q, \alpha\left|\phi_{i}^{2} u\right|^{Q^{\prime}}\right) d \xi & =\int_{B_{h}\left(\xi_{i}, 2 r\right)} \frac{1}{\left|\xi^{\beta}\right|_{h}^{\beta}} \zeta\left(Q, \alpha\left(1+\frac{4}{\tau r}\right)^{Q^{\prime}}\left|\widetilde{u}_{i}\right|^{Q^{\prime}}\right) d \xi \\
& \leq C \int_{B_{h}\left(\xi_{i}, 2 r\right)}\left|\nabla_{\mathbb{H}^{n}} \widetilde{u}_{i}\right|^{Q} d \xi \\
& \leq C \int_{B_{h}\left(\xi_{i}, 2 r\right)}\left|\nabla_{\mathbb{H}^{n}}\left(\phi_{i}^{2} u\right)\right|^{Q} d \xi
\end{aligned}
$$

Combining (4.6) and (4.10), we obtain

$$
\begin{aligned}
\int_{\mathbb{H}^{n}} \frac{1}{|\xi|_{h}^{\beta}} \zeta\left(Q, \alpha|u|^{Q^{\prime}}\right) d \xi & \leq \sum_{i} \int_{B_{h}\left(\xi_{i}, r\right)} \frac{1}{|\xi|_{h}^{\beta}} \zeta\left(Q, \alpha\left|\phi_{i}^{2} u\right|^{Q^{\prime}}\right) d \xi \\
& \leq \sum_{i} \int_{B_{h}\left(\xi_{i}, 2 r\right)} \frac{1}{\mid \xi_{h}^{\beta}} \zeta\left(Q, \alpha\left|\phi_{i}^{2} u\right|^{Q^{\prime}}\right) d \xi \\
& \leq C \sum_{i} \int_{\mathbb{H}^{n}}\left|\nabla\left(\phi_{i}^{2} u\right)\right|^{Q} d \xi .
\end{aligned}
$$


Using the inequality $|a+b|^{Q} \leq 2^{Q}|a|^{Q}+2^{Q}|b|^{Q}, \forall a, b \in \mathbb{R}, 0 \leq \phi_{i} \leq 1$ and (4.8), we get

$$
\begin{aligned}
\int_{\mathbb{H}^{n}}\left|\nabla_{\mathbb{H}^{n}}\left(\phi_{i}^{2} u\right)\right|^{Q} d \xi & \leq 2^{Q} \int_{\mathbb{H}^{n}}\left(\left.\left.\phi_{i}^{2 Q}\right|_{\mathbb{H}^{n} n} u\right|^{Q}+\left|\nabla_{\mathbb{H}^{n}} \phi_{i}^{2}\right|^{Q}|u|^{Q}\right) d \xi \\
& \leq 2^{Q} \int_{\mathbb{H}^{n}} \phi_{i}\left|\nabla_{\mathbb{H}^{n}} u\right|^{Q} d \xi+\left(\frac{8}{r}\right)^{Q} \int_{\mathbb{H}^{n}} \phi_{i}|u|^{Q} d \xi .
\end{aligned}
$$

In view of (4.7), it then follows that

$$
\begin{aligned}
\sum_{i} \int_{\mathbb{H}^{n}}\left|\nabla_{\mathbb{H}^{n}}\left(\phi_{i}^{2} u\right)\right|^{Q} d \xi & \leq 2^{Q} \sum_{i} \int_{\mathbb{H}^{n}} \phi_{i}\left|\nabla_{\mathbb{H}^{n}} u\right|^{Q} d \xi+\left(\frac{8}{r}\right)^{Q} \sum_{i} \int_{\mathbb{H}^{n}} \phi_{i}|u|^{Q} d \xi \\
& \leq 96^{Q} \int_{\mathbb{H}^{n}}\left|\nabla_{\mathbb{H}^{n}} u\right|^{Q} d \xi+\left(\frac{384}{r}\right)^{Q} \int_{\mathbb{H}^{n}}|u|^{Q} d \xi
\end{aligned}
$$

This together with (4.11) implies

$$
\int_{\mathbb{H}^{n}} \frac{1}{|\xi|^{\beta}} \zeta\left(Q, \alpha|u|^{Q^{\prime}}\right) d \xi \leq \widetilde{C}
$$

for some constant $\widetilde{C}$ depending only on $C, Q$, and $r$. Hence we conclude (1.10).

Secondly, we prove that for any fixed $\beta: 0 \leq \beta<Q, \alpha>0$, and $u \in W^{1, Q}\left(\mathbb{H}^{n}\right)$, there holds

$$
\int_{\mathbb{H}^{n}} \frac{1}{|\xi|_{h}^{\beta}} \zeta\left(Q, \alpha|u|^{Q^{\prime}}\right) d \xi<\infty .
$$

Since $C_{0}^{\infty}\left(\mathbb{H}^{n}\right)$ is dense in $W^{1, Q}\left(\mathbb{H}^{n}\right)$, we can take some $u_{0} \in C_{0}^{\infty}\left(\mathbb{H}^{n}\right)$ such that $\left\|u-u_{0}\right\|_{W^{1, Q}\left(\mathbb{H}^{n}\right)}<\epsilon$, where $\epsilon>0$ is a small number to be determined later. Set

$$
w=\frac{u-u_{0}}{\left\|u-u_{0}\right\|_{W^{1, Q}\left(\mathbb{H}^{n}\right)}} .
$$

Then $\|w\|_{W^{1, Q}\left(\mathbb{H}^{n}\right)}=1$. We divide the proof of (4.12) into two cases:

Case 1. $\beta=0$.

Recall [4.1). By ([12], Lemma 2.2), $\zeta(Q, t)$ is convex with respect to $t$. Since $|a+b|^{\gamma} \leq$ $(1+\delta)|a|^{\gamma}+C(\delta, \gamma)|b|^{\gamma}, \forall a, b \in \mathbb{R}, \gamma \geq 1, \delta>0$, for some constant $C(\delta, \gamma)$ depending only on $\delta$ and $\gamma$, we obtain

$$
\begin{aligned}
& \int_{\mathbb{H}^{n}} \zeta\left(Q, \alpha|u|^{Q^{\prime}}\right) d \xi=\int_{\mathbb{H}^{n}} \zeta\left(Q, \alpha\left|u-u_{0}+u_{0}\right|^{Q^{\prime}}\right) d \xi \\
& \leq \int_{\mathbb{H}^{n}} \zeta\left(Q, \alpha(1+\delta)\left|u-u_{0}\right|^{Q^{\prime}}+\alpha C\left(\delta, Q^{\prime}\right)\left|u_{0}\right|^{Q^{\prime}}\right) d \xi \\
& \left.\leq \frac{1}{\mu} \int_{\mathbb{H}^{n}} \zeta\left(Q, \mu \alpha(1+\delta)\left|u-u_{0}\right|^{Q^{\prime}}\right)\right) d \xi+\frac{1}{v} \int_{\mathbb{H}^{n}} \zeta\left(Q, v \alpha C\left(\delta, Q^{\prime}\right)\left|u_{0}\right|^{Q^{\prime}}\right) d \xi
\end{aligned}
$$

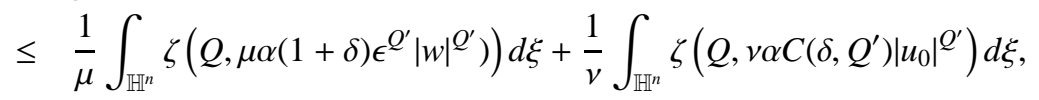

where $1 / \mu+1 / v=1, \mu>1, v>1$. Now we choose $\epsilon>0$ sufficiently small such that $\mu \alpha(1+\delta) \epsilon^{Q^{\prime}}<\alpha_{Q}$. By (1.10), there holds

$$
\left.\int_{\mathbb{H}^{n}} \zeta\left(Q, \mu \alpha(1+\delta) \epsilon^{Q^{\prime}}|w|^{Q^{\prime}}\right)\right) d \xi \leq C_{1}
$$


for some constant $C_{1}$ depending only on $n$ and $\tau$. In addition, since $u_{0} \in C_{0}^{\infty}\left(\mathbb{H}^{n}\right)$, it is obvious that

$$
\int_{\mathbb{H}^{n}} \zeta\left(Q, v \alpha C\left(\delta, Q^{\prime}\right)\left|u_{0}\right|^{Q^{\prime}}\right) d \xi<\infty
$$

Therefore, we have

$$
\int_{\mathbb{H}^{n}} \zeta\left(Q, \alpha|u|^{Q^{\prime}}\right) d \xi<\infty
$$

Case 2. $0<\beta<Q$.

Note that

$$
\int_{\mathbb{H}^{n}} \frac{1}{|\xi|_{h}^{\beta}} \zeta\left(Q, \alpha|u|^{Q^{\prime}}\right) d \xi \leq \int_{|\xi|_{h} \leq 1} \frac{1}{|\xi|_{h}^{\beta}} \zeta\left(Q, \alpha|u|^{Q^{\prime}}\right) d \xi+\int_{\mathbb{H}^{n}} \zeta\left(Q, \alpha|u|^{Q^{\prime}}\right) d \xi .
$$

This together with Hölder's inequality and Case 1 implies (4.12).

Finally, we confirm that for any $\alpha>\alpha_{Q}(1-\beta / Q)$, there holds

$$
\sup _{\|u\|_{1, \tau} \leq 1} \int_{\mathbb{H}^{n}} \frac{1}{|\xi|_{h}^{\beta}} \zeta\left(Q, \alpha|u|^{Q^{\prime}}\right) d \xi=\infty .
$$

This is based on calculations of related integrals of the Moser function sequence. We omit the details but refer the reader to [3].

Acknowledgements. This work was partly supported by the NSFC 11171347 and the NCET program 2008-2011.

\section{References}

[1] Adimurthi, Y. Yang, An interpolation of Hardy inequality and Trudinger-Moser inequality in $\mathbb{R}^{N}$ and its applications, International Mathematics Research Notices 13 (2010) 2394-2426.

[2] D. Cao, Nontrivial solution of semilinear elliptic equations with critical exponent in $\mathbb{R}^{2}$, Commun. Partial Differential Equations 17 (1992) 407-435.

[3] W. Cohn, N. Lam, G. Lu, Y. Yang, The Moser-Trudinger inequality in unbounded domains of Heisenberg group and sub-elliptic equations, Nonlinear Analysis (2011), doi: 10.1016/j.na.2011.09.053.

[4] W. Cohn, G. Lu, Best constants for Moser-Trudinger inequalities on the Heisenberg group, Indiana Univ. Math. J. 50 (2001) 1567-1591.

[5] J. M. do Ó, $N$-Laplacian equations in $\mathbb{R}^{N}$ with critical growth, Abstr. Appl. Anal. 2 (1997) 301-315.

[6] G. Hardy, J. Littlewood, G. Polya, Inequalities, Cambridge University Press, 1952.

[7] E. Hebey, Sobolev spaces on Riemannian maifolds, Lecture notes in mathematics 1635, Springer, 1996.

[8] Y. Li, B. Ruf, A sharp Trudinger-Moser type inequality for unbounded domains in $\mathbb{R}^{N}$, Ind. Univ. Math. J. 57 (2008) 451-480.

[9] R. Panda, Nontrivial solution of a quasilinear elliptic equation with critical growth in $\mathbb{R}^{n}$, Proc. Indian Acad. Sci. (Math. Sci.) 105 (1995) 425-444.

[10] B. Ruf, A sharp Trudinger-Moser type inequality for unbounded domains in $\mathbb{R}^{2}$, J. Funct. Anal. 219 (2005) 340367.

[11] E. M. Stein, Harmonic analysis, Princeton University press, 2006.

[12] Y. Yang, Existence of positive solutions to quasi-linear elliptic equations with exponential growth in the whole Euclidean space, J. Funct. Anal. 262 (2012) 1679-1704, arXiv: 1106.4622v1.

[13] Y. Yang, Trudinger-Moser inequalities on complete noncompact Riemannian manifolds, arXiv: 1112.0724v1. 